\title{
RAPID VARIABILITY OF GAMMA-RAY BLAZARS
}

\author{
M. SALVATI \\ Osservatorio Astrofisico di Arcetri \\ L. E.Fermi 5 I-50125 Firenze Italy \\ M. SPADA \\ Dipartimento di Astronomia e Scienza dello Spazio \\ Università di Firenze \\ L. E.Fermi 5 I-50125 Firenze Italy \\ AND \\ F. PACINI \\ Osservatorio Astrofisico di Arcetri \\ and \\ Dipartimento di Astronomia e Scienza dello Spazio \\ L. E.Fermi 5 I-50125 Firenze Italy
}

\section{Relevant Timescales}

This paper is devoted to a discussion of the rapid variability observed in $\gamma$-ray blazars. We do this in the framework of a widely accepted scenario, according to which the blazar emission arises from a jet, i.e. a continuous flow of relativistic fluid, with perturbations occasionally superimposed. The jet is assumed to have a bulk Lorentz factor $\Gamma \gg 1$, and length $z$ and radius $r$ such that $r \approx z / \Gamma$. The observer's line of sight makes an angle $\theta \approx 1 / \Gamma$ to the jet axis.

The time scales which are relevant to the variability are the following ones:

1. the geometrical time needed to a photon to cross the emission region either in length $\left(\Delta t_{z} \approx\right.$ $\left.z / c / \Gamma^{2}\right)$ or in width $\left(\Delta t_{r} \approx r / c / \Gamma\right)$; because of the assumed geometry, $\Delta t_{z} \approx \Delta t_{r}$

2. the radiative time $\Delta t_{e m}$ over which the emitting electrons lose a large fraction of their energy; if $\gamma$ is the typical electron Lorentz factor in the comoving frame, the dot indicates the time derivative, and the prime refers to quantities measured in the comoving frame, then $\Delta t_{e m} \approx$ $(\gamma / \dot{\gamma})^{\prime} / \Gamma$

3. the injection time $\Delta t_{\text {in }}$ over which the electrons are fed to the emission region; as will be shown in the following, the radiative time is typically shorter than the geometrical time, and the electrons must be accelerated in situ; there are arguments (Blandford and Levinson 1995) which point to the electrons being not only accelerated, but also generated in situ; in any case the original energy carrier -whichever its form- has to come from the center, and $\Delta t_{i n}$-the time scale of its typical variations- must reflect the dimensions of the central engine.

¿From the point of view of the observer, a variability episode appears convolved with all the time scales listed above, and is dominated by the longest one among them. As a partial exception, we note that in the case of a diverging jet the emission mechanisms become very rapidly ineffective with $z$, and the radiative time scale is irrelevant even when $\Delta t_{z}<\Delta t_{e m}$; the efficiency of the jet is drastically reduced, however.

The injection time scale $\Delta t_{i n}$ is very likely the shortest of all, of the order of several minutes, depending on the mass one chooses for the central black hole. The remaining time scales $\Delta t_{e m}$ and $\Delta t_{z}$ are related in an interesting way to the jet optical depth to photon-photon absorption. This is due to the near equality of the cross sections involved, and to the radiation density in the jet being the target for both the emission and the absorption processes. The relation can be expressed in 
closed form under certain assumptions: only the jet contributes to the radiation field, the radiation spectrum is flat in $\nu F_{\nu}$, the scattering process is always in the Thomson regime. Then one has

$$
\ell=\frac{L \sigma_{T}}{m c^{3} z}, \quad \frac{\Delta t_{e m}}{\Delta t_{z}}=\frac{3 \pi}{4 \ell} \frac{\Gamma^{3}}{\gamma}, \quad \tau_{\gamma \gamma}=\frac{3 \ell}{16 \pi} \frac{\epsilon_{u}}{\Gamma^{4}}\left(\ln \frac{\epsilon_{\mathrm{u}}}{\epsilon_{\mathrm{d}}}\right)^{-1}
$$

The quantity $\ell$ is the compactness of the jet radiation field, $\tau_{\gamma \gamma}$ is the jet photon-photon optical depth, and $\epsilon_{u}$ and $\epsilon_{d}$ are the upper and lower energies of the photon spectrum, respectively, in units of $m c^{2}$. By multiplying Eqs. (1b) and (1c) we finally get

$$
\frac{\Delta t_{e m}}{\Delta t_{z}} \tau_{\gamma \gamma}=\frac{0.14}{\ln \frac{\epsilon_{u}}{\epsilon_{\mathrm{d}}}} \frac{\epsilon_{u}}{\gamma \Gamma}
$$

As long as the right hand side is $\ll 1$, one can have at the same time $\tau_{\gamma \gamma} \leq 1$ and a high radiative efficiency. In certain BL Lacs, however, as for instance Mkn 421, the Klein-Nishina regime is reached and $\epsilon_{u} / \gamma \Gamma \approx 1$; furthermore, if one requires that the jet is not loaded with too many pairs (Sikora et al. 1997; Ghisellini \& Madau 1996), $\tau_{\gamma \gamma}$ has to be $\ll 1$, and a certain degree of fine tuning becomes necessary. In general, however, $\Delta t_{e m}<\Delta t_{z}$, and the minimum variability time scale is determined by the geometry of the jet. The models which have been fitted to typical $\gamma$-ray blazars have by construction $\Delta t_{z}$ equal to the observed variability time scale, which is several hours; Salvati et al. (1996), for instance, in the case of $3 \mathrm{C} 279$ find $\Gamma \approx 10$ and $z \approx 10^{17} \mathrm{~cm}$.

A special problem is posed by the $\mathrm{TeV}$ light curve of Mkn 421, which in May 1996 exhibited a very rapid flare with rise and decay times of only several minutes (Gaidos et al. 1996). If one insists that the variability is governed by $\Delta t_{z}$, then one must require $\Gamma \gg 10$, or $z \ll 10^{17} \mathrm{~cm}$, or both. We instead propose a scenario which can accomodate very rapid flares even if the jet geometry and $\Delta t_{z}$ are the same as in $3 \mathrm{C} 279$; it is found that the repetition time of the flares is limited by $\Delta t_{z}$, but the flare duration is not.

\section{The Geometry of the Emission Region}

The acceleration of the emitting electrons must occur in situ, and strong shocks are the most likely location. The orientation of the shocks with respect to the jet axis is preferentially at angles $\approx 1 / \Gamma$. This is seen in numerical simulations (Bowman 1994; Gómez et al. 1997), and is due to arcsin $1 / \Gamma$ being the causal angle traced by a perturbation which moves isotropically at speed $c$ in the comoving frame. Also, when the flow is deflected by the external medium, angles around $1 / \Gamma$ are preferred, since at those angles the ram pressure levels off at values close to the internal pressure. Finally, $\Delta t_{i n}$ and $\Delta t_{e m}$ are much smaller than $\Delta t_{z}$ (this remains true if we use the luminosity of Mkn 421 within the geometry of $3 \mathrm{C} 279$, since the lower luminosity is compensated almost exactly by the higher $\gamma$ ).

We can envisage a geometry of the emission region similar to Fig. 1: the accelerating shocks are a series of opening and closing cones; a very thin layer of extra electrons (the perturbation) move down the jet with velocity $\beta c, \beta=\sqrt{1-1 / \Gamma^{2}}$; they radiate immediately after being accelerated, so that the emission region is a ring at the intersection between the electron layer and the conical shocks; if the cone opening is precisely $\arcsin 1 / \Gamma=\operatorname{arcso} \beta$, and the line of sight coincides precisely with one generatrix of the cone, the photons emitted in the observer's direction pile up at the same observed time.

Many of these restrictions can be released. One can assume that the cone opening angle $\alpha$ and the viewing angle $\theta$ are different from each other; then the pile up condition becomes

$$
\frac{\beta c}{\cos \alpha}=\frac{c}{\cos (\theta-\alpha)}, \quad \theta=\alpha \pm \operatorname{arcos} \frac{\cos \alpha}{\beta}
$$

When $\alpha>\operatorname{arcos} \beta$, the pile up effect takes place along two lines of sight. Also, it is not essential that $\theta$ coincides exactly with the pile up value. Figure $2 a$ shows the result of viewing angles differing by various amounts from the optimal one. One should note that the vertical axis is logarithmic, and the height of the spikes is very often a factor of several; one should further note that blazars are observationally selected to have $\theta \leq \operatorname{arcos} \beta$, and the $a$ posteriori probability of having $\theta / \operatorname{arcos} \beta \geq x$ 


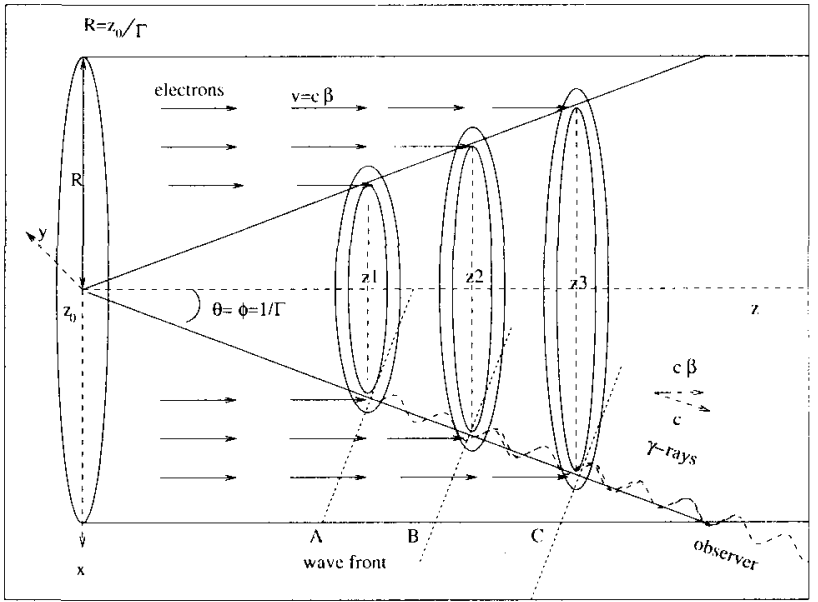

Figure 1. The geometry assumed for the acceleration and emission region. See text for details.
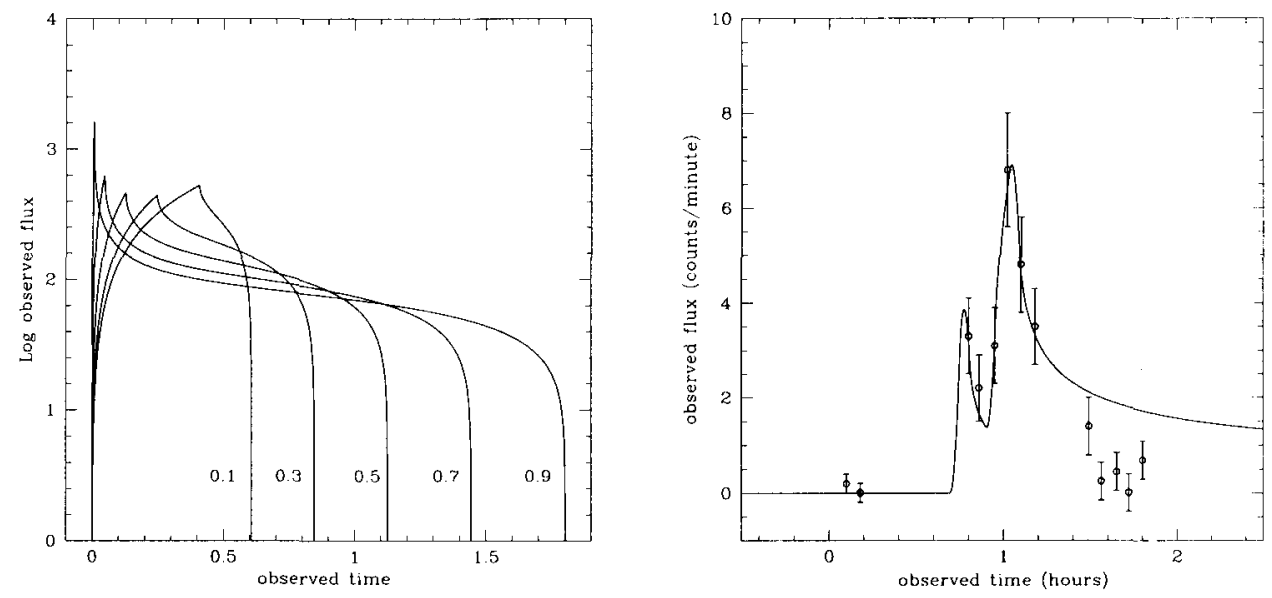

Figure 2. Panel a: The light curves observed for $\alpha=\operatorname{arcos} \beta$ and $x=\theta / \operatorname{arcos} \beta \neq 1$. The values of $x$ are indicated by the labels.

Panel b: A fit to the TeV flare of $\mathrm{Mkn} 421$. The geometrical time scale of the underlying model is $\Delta t_{z}=9$ hours.

is relatively large, $1-x^{2}$; for instance, the sharpest peak shown in Fig. 2a should be observable in $19 \%$ of the cases. Other deviations from the ideal geometry could be some curvature in the shocks, or a non-negligible lifetime of the electrons at low energies; these should result in a certain amount of smoothing, which needs to be computed in detail.

Within the present assumptions the curves of Fig. 2a are the Green functions of the variability problem, and by convolving them with an arbitrary injection function one obtains the general solution. Figure $2 \mathrm{~b}$ is one example, fitted to the TeV flare of Mkn 421. 


\section{Discussion}

The sharp features of our Green functions carry only a small fraction of the total energy of the perturbation; most of this energy resides in the long lived, low level tail, which by construction has a time scale of order $\Delta t_{z}$. So the observed light curve will exhibit conspicuous sharp features only if the injection events are spaced at intervais $\geq \Delta t_{z}$; in the opposite case a given peak will be swamped by the superposition of the many tails of the preceding peaks.

The peaks appear in the Green functions because the relevant geometry is not the one of the jet as a whole, but instead the one of the emission region, which -in the case of strong radiation losses- traces the surface of the shocks. The preferential orientation of this surface results in the radiation field being strongly anysotropic at any point of the emission region at the time of the perturbation; the peak photons are distributed over an angle much narrower than $1 / \Gamma$, of the order of $(1 / \Gamma) \times \sqrt{\Delta t_{e m} / \Delta t_{z}}$, and from the point of view of the variability time scales this entails the same compression of an "effective" Lorentz factor $\approx \Gamma \times \sqrt{\Delta t_{z} / \Delta t_{e m}}$.

Anisotropies in the laboratory frame on scales smaller than $1 / \Gamma$ are equivalent to the loss of isotropy in the comoving frame, at variance with the standard scenario, and may have interesting consequences. While the peak photons, as we have found, are very closely aligned, the emitting electrons interact with the same peak photons at angles of the order of $1 / \Gamma$; this breaks the assumptions under which Eq. 2 was derived, and allows a situation where at the same time the radiative losses are very rapid and the pair production opacity is very low. Also, if the line of sight is not closely aligned with the shock surface, the observer will infer a photon density at the source lower than the one experienced by the electrons; this breaks the assumptions under which synchrotron self Compton models are usually computed, and allows larger ratios between the Compton and synchrotron regions of the spectrum. For instance, the 1991 high state of $3 \mathrm{C} 279$ could not be fitted without assuming extra target photons, due either to an external source or to an ad hoc comoving-frame anisotropy (Hartman et al. 1996).

The considerations above are very preliminary, and many side effects have to be investigated in detail. The photons in the low level tail of the perturbation are again isotropic in the comoving frame, and since they carry most of the energy many conclusions relevant to the initial part of the outburst may become inapplicable at later times. As we have seen, Eq. 2 is invalid during the peak, but it resumes validity afterwards, and one could observe a time-dependent pair production cutoff in the spectrum. More realistic models must be computed in order to assess these points.

\section{Acknowledgements}

This work was partly supported by the Italian Space Agency through grants ASI-95-RS-120 and ARS-96-66.

\section{References}

Blandford, R.D., \& Levinson, A. 1995, ApJ 441, 79

Bowman, M. 1994, MNRAS 269, 137

Gaidos, J.A. et al. 1996, Nature 383, 319

Ghisellini, G., \& Madau, P. 1996, MNRAS 280, 67

Gómez, J.L., Martí, J.M., Marscher, A.P., Ibáñez, J.M., \& Alberdi, A. 1997, ApJLetters 482, L33

Hartman, R.C. et al. 1996, ApJ 461, 698

Salvati, M., Spada, M., \& Pacini, F. 1996, in Gamma-ray Emitting AGN, ed. J.G. Kirk, M. Camenzind, C. von Montigny \& S. Wagner (Heidelbergh: MPI für Kernphysik V37-96), 161

Sikora, M., Madejski, G., Moderski, R., \& Poutanen, J. 1997, ApJ 484, 108 